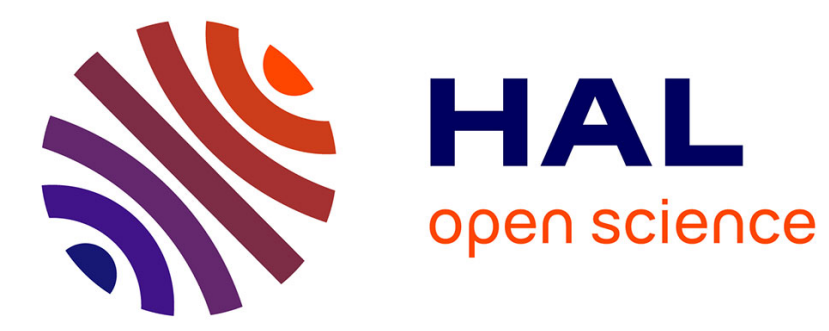

\title{
Metallic Magnetic Calorimeters for Absolute Activity Measurement
}

Martin Loidl, Elvire Leblanc, Matias Rodrigues, Jacques Bouchard, B. Censier, Thierry Branger, D. Lacour

\section{- To cite this version:}

Martin Loidl, Elvire Leblanc, Matias Rodrigues, Jacques Bouchard, B. Censier, et al.. Metallic Magnetic Calorimeters for Absolute Activity Measurement. Journal of Low Temperature Physics, 2008, 151 (3-4), pp.1055-1060. 10.1007/s10909-008-9793-7 . cea-02475514

HAL Id: cea-02475514 https://hal-cea.archives-ouvertes.fr/cea-02475514

Submitted on 13 Feb 2020

HAL is a multi-disciplinary open access archive for the deposit and dissemination of scientific research documents, whether they are published or not. The documents may come from teaching and research institutions in France or abroad, or from public or private research centers.
L'archive ouverte pluridisciplinaire HAL, est destinée au dépôt et à la diffusion de documents scientifiques de niveau recherche, publiés ou non, émanant des établissements d'enseignement et de recherche français ou étrangers, des laboratoires publics ou privés. 


\title{
Metallic magnetic calorimeters for absolute activity measurement
}

\author{
M. Loidl, E. Leblanc, M. Rodrigues, J. Bouchard, B. Censier, \\ T. Branger, D. Lacour
}

CEA Saclay, DRT/LIST/DETECS/LNHB, 91191 Gif-sur-Yvette, France

We present a prototype of metallic magnetic calorimeters that we are developing for absolute activity measurements of low energy emitting radionuclides. We give a detailed description of the realization of the prototype, containing an ${ }^{55} \mathrm{Fe}$ source inside the detector absorber. We present the analysis of first data taken with this detector and compare the result of activity measurement with liquid scintillation counting. We also propose some ways for reducing the uncertainty on the activity determination with this new technique.

PACS numbers: 29.40.-n; 29.40Vj; 06.20.+v

\section{INTRODUCTION}

One of the tasks in the field of ionising radiation metrology is absolute activity measurement, i. e. the determination of the mass activity of a given radioactive solution. Ideally this determination should not depend on detection efficiency calibration or on the knowledge of the solid angle or of the decay scheme. This would require a $4 \pi$ detection geometry and a $100 \%$ detection efficiency. In many cases the detectors that are commonly in use for activity measurement (semiconductor detectors, liquid or solid scintillators, ionisation chambers, and sometimes a combination of two of them) cannot fulfil these two requirements at the same time. Efficiency calibrations and corrections for the solid angle and for the non-detected fraction of radiation contribute to the uncertainty on the activity determination.

We are developing metallic magnetic calorimeters ${ }^{1,2}$ for activity measurements. The absorber encloses the source to be measured in a $4 \pi$ geometry and we aim to approach a detection efficiency of $100 \%$ as closely as possible. The advantage we see in magnetic calorimeters is that they can offer a high detection efficiency associated with a low detection threshold. To validate this new method we have chosen ${ }^{55} \mathrm{Fe}$, a low energy emitting nuclide whose theoretical emission spectrum is quite well known. 


\section{Loidl et al.}

\section{TOTAL EMISSION SPECTRUM OF ${ }^{55} \mathrm{Fe}$}

${ }^{55} \mathrm{Fe}$ decays by electron capture on the $\mathrm{K}, \mathrm{L}, \mathrm{M}$ and $\mathrm{N}$ shells with respective probabilities of $88.53 \%, 9.83 \%, 1.57 \%$ and $0.06 \%$. Each capture is followed by an electronic rearrangement associated with the emission of a cascade of X-rays and/or Auger electrons. The total energy of the emission cascade for each capture corresponds to the binding energy of the captured electron: the total emission spectrum exhibits lines at $6.539 \mathrm{keV}$ for $\mathrm{K}$ capture, $769 \mathrm{eV}$ for capture on the $\mathrm{L}_{1}$ shell (capture on the $\mathrm{L}_{2}$ shell is strongly suppressed), and a complex of lines in the range $3 \mathrm{eV}$ to $84 \mathrm{eV}$ for capture on the $\mathrm{M}$ sub-shells. Calculations as well as a previous feasibility study $^{3}$ indicate that $\mathrm{M}$ capture occurs mainly on the $\mathrm{M}_{1}$ sub-shell $(84 \mathrm{eV})$.

\section{A PROTOTYPE DETECTOR FOR THE ACTIVITY MEASUREMENT OF ${ }^{55} \mathrm{Fe}$}

In the above mentioned feasibility study we have demonstrated that a detection efficiency close to $100 \%$ for electrons and photons up to $6.5 \mathrm{keV}$ and a very low detection threshold (around $20 \mathrm{eV}$ ) can be realised with metallic magnetic calorimeters. Now we have realised a prototype magnetic calorimeter for activity measurement with an ${ }^{55} \mathrm{Fe}$ source contained in a gold absorber. Monte Carlo simulations have been made in order to determine the thickness of gold required to absorb $99.9 \%$ of all electrons and photons (maximum energy $6.5 \mathrm{keV}$ ) emitted by the source. The simulations were performed using the PENELOPE code which is particularly well suited for low energy radiations.

For activity measurement, the mass of solution used to realise the source must be determined. We used a solution whose mass activity had previously been determined by liquid scintillation counting. This was necessary to have a reference value to compare with the result of the calorimeter measurement. A small drop of this solution was deposited on a $12 \mu \mathrm{m}$ thick gold foil on which a hydrophobic agent had previously been applied in a circular shape to prevent the drop to flow up to the edge of the foil. The drop was weighed $(m=(253 \pm 8) \mu \mathrm{g})$ with a precision balance.

After the solution has dried, the foil was folded, pressed and diffusion welded to enclose the source and assure a good thermal contact within the absorber. The final size of the absorber is $0.70 \mathrm{~mm} \mathrm{x} 1.56 \mathrm{~mm} \times$ ( $2 \times 12) \mu \mathrm{m}$. It is a compromise between a size of the foil large enough to be able to deposit a drop of solution that can be weighed with a reasonably low uncertainty, and a heat capacity small enough to obtain a low detection 


\section{Metallic magnetic calorimeters for absolute activity measurement}

threshold. This absorber is much bigger (a factor 260 in volume) than the one of the feasibility study where we hadn't aimed for determining the mass of the source. Correspondingly the detection threshold was not as low (one order of magnitude higher).

The thermometer is a cuboid $(50 \mu \mathrm{m} \times 50 \mu \mathrm{m} \times 15 \mu \mathrm{m})$ of gold doped with erbium (mass concentration $0.9 \%$ ). It was fixed to the absorber by wedge bonding, thus assuring a metallic contact and a very efficient heat transfer. The detector (absorber + thermometer) was placed on the SQUID chip, with the thermometer being placed inside the SQUID sensing loop. The thermal link consists in a gold wire (diameter $25 \mu \mathrm{m}$, length $1 \mathrm{~mm}$ ) wedge bonded to the absorber and to the copper detector holder.

We are using a two-stage SQUID setup with the first SQUID (SQUID 1) reading out the thermometer and the second one (SQUID 2) acting as a first stage amplifier for the signal delivered by SQUID 1.

Figure 1 represents a cross section of the detector (top) and the twostage SQUID setup (bottom).

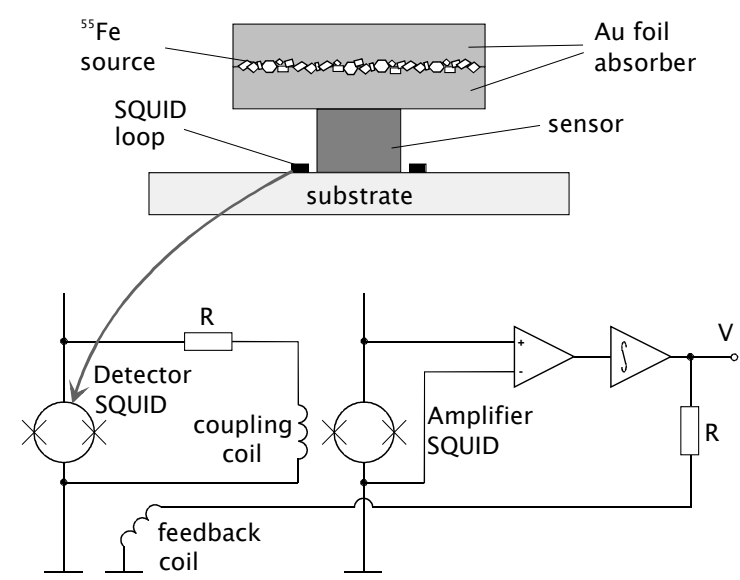

Figure 1: Schematic cross-section of the detector used in the present work. The lower part of the figure represents the two-stage SQUID readout system.

The detector integrated with SQUID 1 was mounted in a dilution refrigerator. The detector temperature was regulated at $16 \mathrm{mK}$ with a standard deviation $\sigma= \pm 1 \mu \mathrm{K}$. SQUID 2 is placed on a dedicated stage of the refrigerator and operated around $3 \mathrm{~K}$. This temperature was also regulated in order to have a stable gain. The magnetic field is initially created with a superconducting coil and then maintained by a superconducting lead ring without injection of an electric current from 


\section{Loidl et al.}

outside the cryostat. This avoids parasitic heating of the cryostat and allows keeping the magnetic field very stable.

\section{FIRST MEASUREMENT AND COMPARISON WITH LIQUID SCINTILLATION COUNTING}

We acquired first data from the prototype detector with a digital oscilloscope at a sampling frequency of $250 \mathrm{kHz}$. For anti-aliasing, the output voltage of the SQUID electronics was low-pass filtered at $100 \mathrm{kHz}$. Using an external clock for triggering, we acquired a sequence of $2 \mathrm{~s}$ time windows, totalling $5000 \mathrm{~s}$ acquisition time. Off-line signal processing was used to detect and count the pulses. The signal was strongly differentiated in order to avoid pile-up. We have chosen this mode of acquisition and pulse detection because triggering on the pulses for acquisition can introduce a bias in the counting which is incompatible with the requirements of metrology.

The pulses have a very long decay time of $70 \mathrm{~ms}$. This was due to a bad bonding contact, as has been found after the measurement. A new detector realised for a background measurement (identical geometry, but without any source inside the absorber) has a decay time constant of $130 \mu \mathrm{s}$.

An energy spectrum (fig. 2) has been established with this data set. The level of the software trigger, set just above the noise, corresponds to an energy of $350 \mathrm{eV}$. The spectrum, containing 12470 events, exhibits two populations of events corresponding to $\mathrm{K}$ and $\mathrm{L}$ capture. The energy of the $\mathrm{M}$ capture events is below threshold.

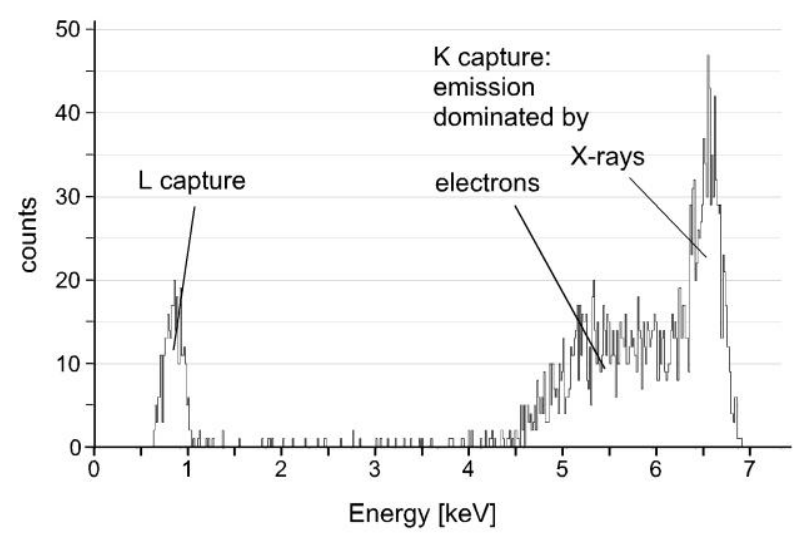

Figure 2: Spectrum of an ${ }^{55} \mathrm{Fe}$ source enclosed in the absorber of a metallic magnetic calorimeter in a $4 \pi$ detection geometry. 


\section{Metallic magnetic calorimeters for absolute activity measurement}

The population representing $\mathrm{K}$ capture presents a peak having a width at half maximum of about $360 \mathrm{eV}$, and a distribution of events extending to lower detected energies. We suppose that the peak corresponds to events where the emission is dominated by a K X-ray, and the large distribution to events dominated by Auger electron emission. This assumption is confirmed by an estimate of the fraction of events corresponding to K X-rays. We find 0.33 , very close to the literature value of the fluorescence yield $\left(\omega_{\mathrm{K}}=0.321 \pm 0.005\right)$. An additional confirmation is provided by the analysis of the signal rise time. Events dominated by a $\mathrm{K} \mathrm{X}$-ray have rise times $(10-$ $90 \%$ ) between 7 and $10 \mu \mathrm{s}$. The X-ray photons interact in the gold foil absorber. The rise time is in this case essentially given by the diffusion of heat from the absorber to the thermometer. Events dominated by Auger electron emission have longer rise times ranging from 20 to $130 \mu \mathrm{s}$. Electrons are mostly absorbed in the crystals forming the source. In that case, phonons created in the source crystals must be transmitted to the gold absorber and couple to the conduction electrons. This energy transfer is relatively slow, leading to longer rise times. The reason for the apparent loss of energy for these events is not yet clear. A phenomenon which may explain that a substantial fraction of the energy of a particle interacting in an insulator (or semiconductor) is not detected is the creation of meta-stable states like trapped electron-hole pairs ${ }^{4}$.

The determination of the pulse heights of $L$ capture events, just above the noise level, is not very precise but largely sufficient to identify them as L captures. The rise times are also difficult to determine, but they roughly correspond to those of $\mathrm{K}$ captures dominated by Auger electron emission. This is in agreement with the fact that only very few L captures $(0.5 \%)$ are followed by X-ray emission. The detected L captures represent, after correction for the non-detected $\mathrm{M}$ and $\mathrm{N}$ captures, a fraction of $0.1019 \pm 0.0029$ of the disintegrations. This is consistent, within the uncertainties, with the capture probability for the $\mathrm{L}$ shell $\left(P_{\mathrm{L}}=0.0983 \pm 0.0013\right)$.

For the activity determination, we have corrected for the dead-time that is applied in the signal processing to avoid pile-up. Taking into account the capture probabilities for the $\mathrm{K}$ and $\mathrm{L}$ shells (i. e. correcting for the nondetection of $\mathrm{M}$ and $\mathrm{N}$ captures), the measured value of the activity of the source is $(2.54 \pm 0.02) \mathrm{Bq}$. Using the mass of the drop of solution deposited on the absorber foil and the recommended half life of ${ }^{55} \mathrm{Fe}$ $\left(t_{1 / 2}=(1003.3 \pm 2.9) \mathrm{d}\right)$, the activity measured with this magnetic calorimeter corresponds to a mass activity of the solution of $(12.4 \pm 0.4) \mathrm{kBq} / \mathrm{g}$. The uncertainty is largely dominated by the uncertainty associated with the mass determination. We have compared this result with the mass activity 


\section{Loidl et al.}

determined by liquid scintillation counting, equal to $(12.641 \pm 0.063) \mathrm{kBq} / \mathrm{g}$. Both values are consistent within the uncertainties.

\section{CONCLUSIONS AND PERSPECTIVES}

We have realised and characterised a first prototype of a new class of detectors that we are developing for absolute activity measurement. This is especially interesting as there are not many techniques available in this field. For absolute activity measurements, it is advantageous to compare results of measurements based on different physics principles in order to unveil possible systematic errors due to one or the other method.

The detectors commonly used for ${ }^{55} \mathrm{Fe}$ activity measurement detect only part of the $\mathrm{K}$ capture events and no $\mathrm{L}$ capture events. With liquid scintillators one can detect roughly $60 \%$ of the K-captures and with X-ray semiconductor detectors $32 \%$ of the K-captures. Our prototype cryogenic detector, although not optimized, has a detection efficiency higher than $99 \%$ for $\mathrm{K}$ and $\mathrm{L}$ captures.

One weak point of the present detector is the fact that the size of the thermometer is far from optimal for the absorber size. Calculations of the signal-to-noise ratio of magnetic calorimeters show that the heat capacity of the thermometer should be equal to that of the absorber. However, our thermometer is limited in size by the diameter of the sensitive loop of the SQUID magnetometer.

The most severe limitation of this detector for activity measurement was the small mass of the source and the uncertainty associated with its determination. For conceiving the prototype detector, we have estimated the maximum absorber heat capacity allowing the $\mathrm{L}$ capture events to be above the detection threshold. Together with the required thickness of gold, this has limited the surface of the absorber foil and hence the size of the drop of radioactive solution. In order to reduce the uncertainty on the mass, we should increase the surface of the absorber foil. There are different ways to make a larger absorber compatible with a low detection threshold.

An obvious idea is to use an absorber material with a lower specific heat than gold but a similar stopping power. This could be a semi-metal like bismuth. Several groups working with bismuth have observed interdiffusion between bismuth and gold ${ }^{5}$, which is the main constituent of our magnetic thermometer. This problem was recently solved by electroplating both gold and bismuth layers ${ }^{6}$. Another natural choice for an absorber material would be a superconductor with a high atomic number, such as tantalum or rhenium. In the present configuration, with the absorber on top of the thermometer and an external field coil, the superconductor would strongly suppress the magnetic field required to magnetise the thermometer. 


\section{Metallic magnetic calorimeters for absolute activity measurement}

Another idea is to replace the small sensor placed directly in the SQUID loop by a thin sensor foil coupled to the readout SQUID via a flux transformer with a meander type pick-up coil ${ }^{2}$. This would permit on the one hand to match the heat capacity of absorber and thermometer, hence to read out larger absorbers, and on the other hand to avoid the difficulty of magnetic field suppression by a superconducting absorber. The thermometer is in this case a thin (few $\mu \mathrm{m}) \mathrm{Au}$ :Er foil covering the surface of the meander structure. The adaptation of the thermometer heat capacity consists in defining the surface of meander and Au:Er foil. We have already used this scheme in two detectors. One of them was developed in collaboration with the University of Heidelberg for beta spectrometry and includes also the source $\left({ }^{36} \mathrm{Cl}\right)$ inside the absorber ${ }^{7}$. The second one was developed in our group for hard X-ray and gamma spectrometry ${ }^{8}$. Both of these detectors have gold absorbers. The group in Heidelberg is testing metallic magnetic calorimeters with meander type readout and superconducting rhenium absorbers ${ }^{9}$. Our next generation of detectors for activity measurement will also be based on this readout scheme, but in a first stage we will use gold absorbers. Superconducting absorbers may be an option for a third generation.

\section{REFERENCES}

1. C. Enss et al., J. Low Temp. Phys. 121, 137-176 (2000)

2. A. Fleischmann, C. Enss and G. Seidel, "Metallic Magnetic

Calorimeters", in Cryogenic Particle Detectors, Topics Appl. Phys. 99, C.

Enss (Ed.), Springer Berlin/Heidelberg, 151 - 216 (2005)

3. M. Loidl, E. Leblanc, T. Branger, H. Rotziner, T. Daniyarov, M. Linck,

A. Fleischmann and C. Enss, Nucl. Instr. \& Meth. A 520, 73 (2004)

4. D. McCammon, M. Juda, J. Zhang, R. L. Kelley, S. H. Moseley and

A. E. Szymkowiak, IEEE Trans. Nucl. Sci. 33, 236 (1985)

5. see e. g. J. E. Sadleir et al., Nucl. Instr. \& Meth. A 559, 447 (2006)

6. S. Bandler et al., these proceedings

7. H. Rotzinger, M. Linck, A. Burck, M. Rodrigues, M. Loidl, E. Leblanc, L. Gastaldo, A. Fleischmann and C. Enss, these proceedings

8. M. Rodrigues, M. Loidl, E. Leblanc, J. Bouchard, B. Censier, A.

Fleischmann, A. Burck, H. Rotzinger and C. Enss, these proceedings 9. J.-P. Porst, C. Höhn, D. Haug, R. Weldle, G. M. Seidel, L. Gastaldo, A.

Fleischmann and C. Enss, these proceedings 